\title{
Quark y la importancia de la voluntad en la disulgación
}

\author{
Edgar Ramos* \\ Miguel García** \\ Bertha Michel*** \\ Viridiana Esparza****
}

Pese a los grandes avances de los últimos veinte años, los recursos de las instituciones de divulgación

en México son insuficientes. En este contexto el trabajo con voluntarios es una opción viable para impulsar la formación de divulgadores, aumentar la comunicación pública de la ciencia y tecnología y, al mismo tiempo, formar divulgadores profesionales. Quark es un grupo de divulgación científica que - desde su origen - ha trabajado, crecido y producido de forma poco usual: el enfoque recreativo es el eje del trabajo a partir del cual se forman sus integrantes. En los últimos doce años la trayectoria del grupo, basada en el trabajo voluntario, observa un crecimiento sostenido. Incluso, algunos integrantes se han convertido en divulgadores profesionales.

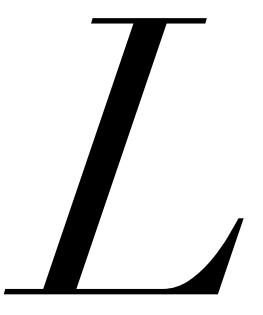
prende las actividades de comunicación que permiten a personas no expertas acceder a la ciencia y la tecnología (CyT), sobre todo a los conocimientos, pero también a la historia, formas de trabajo, conflictos y retos. No se trata de "iluminar" al público con la sabiduría de un investigador, profesor o comunicador. La divulgación procura establecer un diálogo para acercar los elementos científicos a la realidad cotidiana del público interesado tomando en cuenta su contexto, intereses, ideas previas y preocupaciones: "la divulgación científica selecciona, redirige, adapta, recrea, un conocimiento producido en el ámbito especializado de ciertas comunidades científicas y tecnológicas para que una vez transformado cumpla una función social dentro de un contexto distinto y con propósitos diferentes para una determinada comunidad cultural".'

* Miembro del Grupo Quark, Museo de Ciencias, Universidad Autónoma de Zacatecas, México. Correo electrónico: edgar@grupoquark.com

** Estudiante del Doctorado en Estudios del Desarrollo y miembro del Grupo Quark, Museo de Ciencias, Universidad Autónoma de Zacatecas, México. Correo electrónico: miguel@grupoquark.com

*** Miembro del Grupo Quark, Museo de Ciencias, Universidad Autónoma de Zacatecas, México. Correo electrónico: tita@grupoquark.com

*** Miembro del Grupo Quark, Museo de Ciencias, Universidad Autónoma de Zacatecas, México. Correo electrónico: frijolito@grupoquark.com 


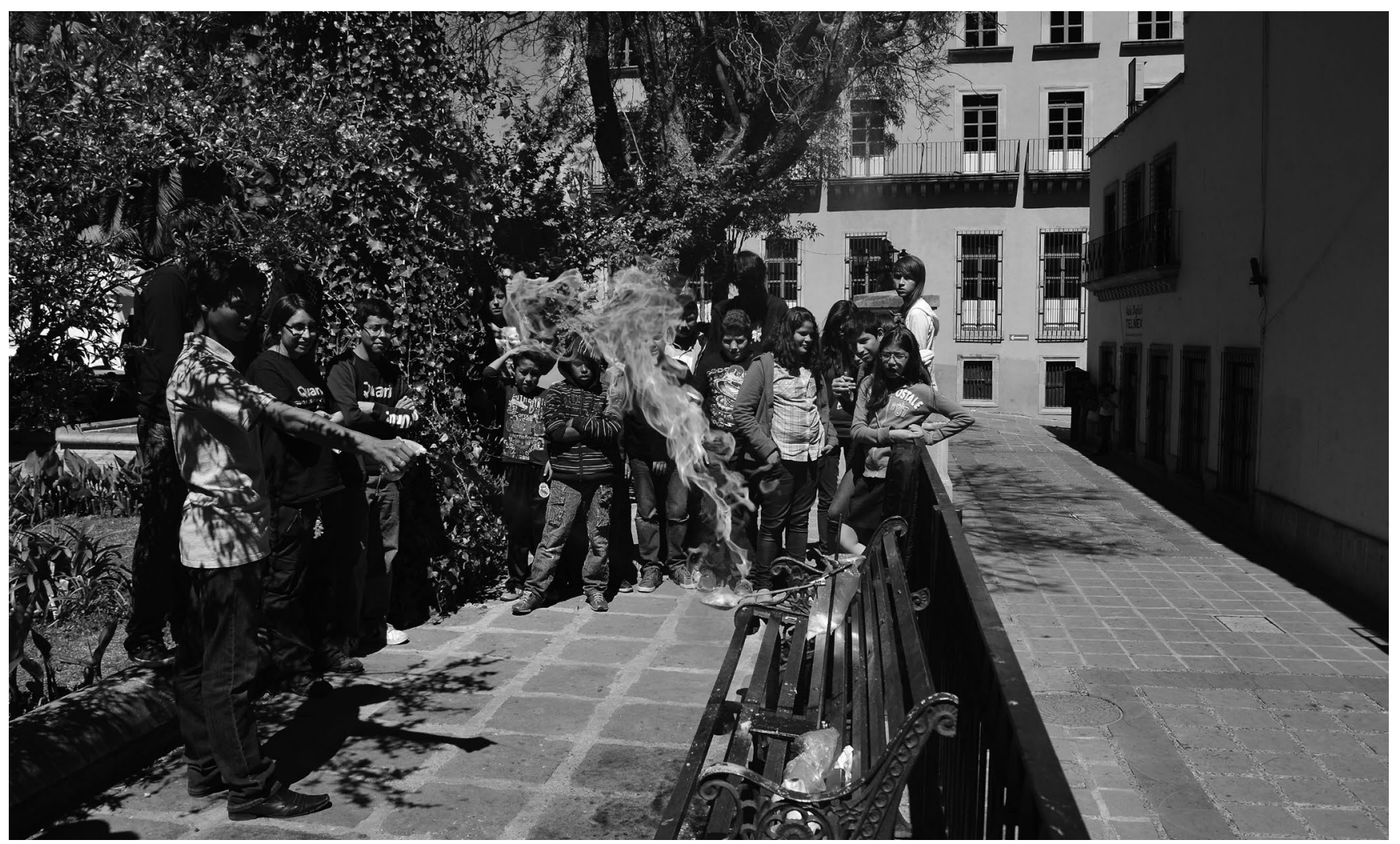

Integrantes del club infantil de la ciencia del Grupo Quark realizan experimentos al aire libre.

Este proceso implica una doble recreación: a) reconstruir en un ámbito no especializado los conocimientos generados por la cyт, y b) despertar el placer, diversión y emoción que las actividades de divulgación pueden incitar en las personas involucradas.

Además de motivar el gusto por la cyт, lo cual no es poca cosa, la divulgación tiene importantes funciones sociales que cumplir, como difundir entre la sociedad los avances científico-tecnológicos más relevantes, abordar las implicaciones sociales que estas novedades puedan traer consigo, fomentar el desarrollo de vocaciones científicas e incluso permitir a los investigadores acercarse al contexto cotidiano. En última instancia, la divulgación le ofrece a los ciudadanos, como individuos y a la sociedad en su conjunto, las herramientas para tomar decisiones en un mundo dominado por los avances de la cyт.

El Museo de Ciencias de la Universidad Autónoma de Zacatecas es una institución pionera en materia de divulgación científica en el estado. Abrió sus puertas al público en octubre de I983 y rápidamente se estableció como un referente para las actividades científicas dirigidas al público no especializado. Si bien desde su fundación se desplegó un esfuerzo considerable por ofertar actividades diversas, los programas llevados a cabo se vieron limitados por el reducido número de personas contratadas para trabajar en el Museo. Aún así, se establecieron las bases para varios programas que siguen activos hoy en día.

A partir de $199^{5}$, jóvenes voluntarios colaboraron en el desarrollo del Club Infantil de la Ciencia. Sin embargo, la labor de este primer equipo de estudiantes no logró fortalecerse lo suficiente como para incidir significativamente en los programas del museo y en el número de personas que atendían. No pudieron consolidarse procesos importantes como la atracción y formación de recursos humanos o el impulso de nuevas actividades.

En septiembre de 200I, por iniciativa del ingeniero Antonio Villarreal Álvarez, se creó Quark, un grupo de divulgación científica especializado en talleres de ciencia recreativa. Desde entonces trabaja de forma conjunta con el Museo de Ciencias. El primer objetivo del grupo fue reactivar el Club Infantil de la Ciencia, que había dejado de funcionar durante un semestre. Hoy, a casi i3 años de su génesis, Quark ha rebasado con creces su meta inicial. Está creciendo considera- 


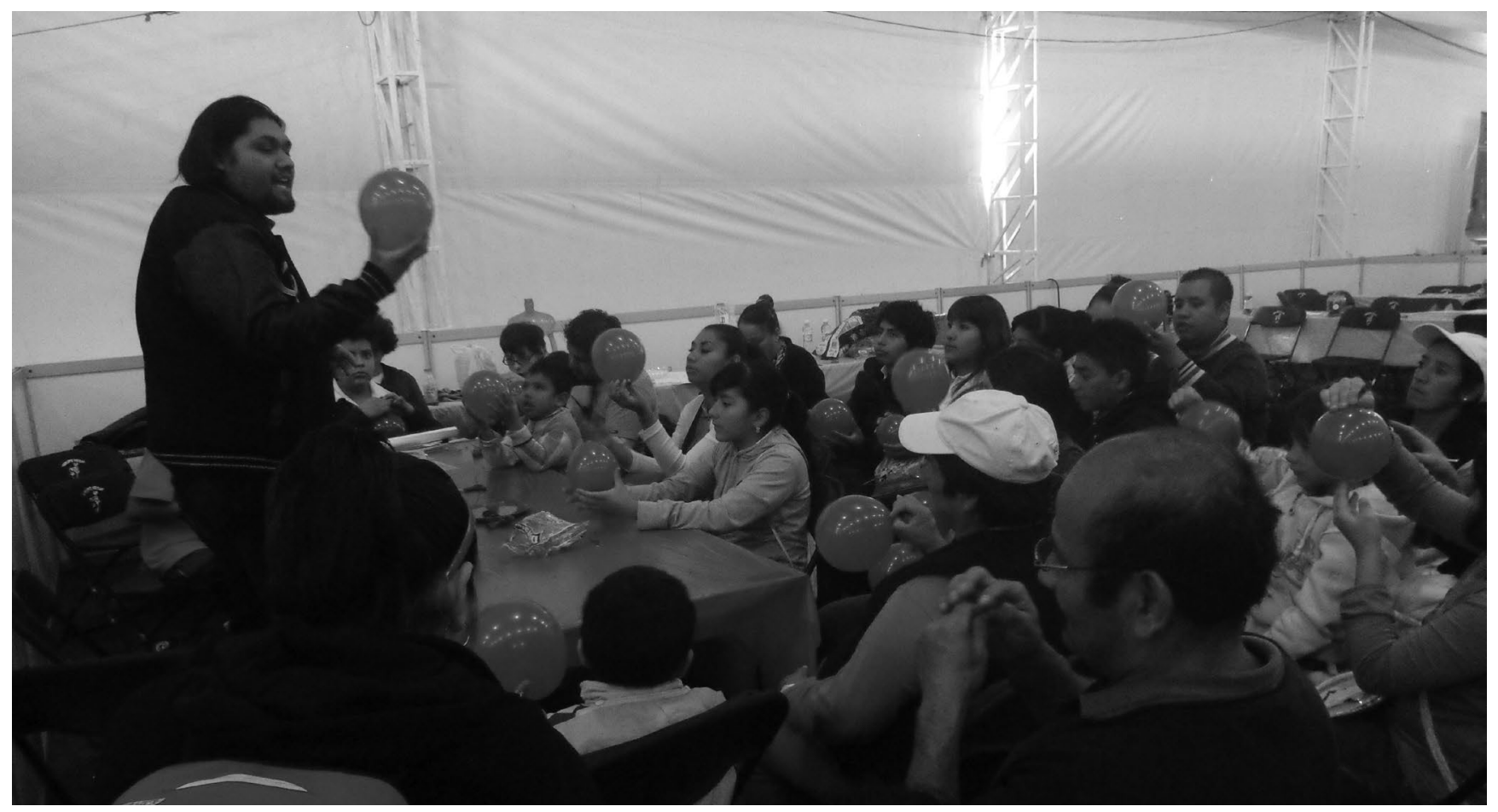

Los divulgadores de la ciencia del Grupo Quark imparten curso y talleres a estudiantes de primaria.

blemente tanto en número de integrantes como de actividades realizadas.

Durante su existencia, el Grupo Quark ha realizado talleres de divulgación científica en 22 de los 58 municipios de Zacatecas y ha brindado atención a más de 5o,ooo personas de forma directa. Además, desde 2002 ha participado de manera ininterrumpida en el Encuentro Nacional de Divulgación Científica, evento organizado por la Sociedad Mexicana de Física; y desde 2004, en la Semana Nacional de Ciencia y Tecnología a cargo del Consejo Nacional de Ciencia y Tecnología (Conacyt). En estos y otros eventos, Quark ha trabajado con otras 6o,ooo personas en i6 estados del país.

En el ámbito académico, miembros del Grupo Quark han presentado más de 40 ponencias y conferencias en eventos nacionales e internacionales de primer nivel. En 2013, junto con el Museo de Ciencias de la UAz, se organizó la Reunión de la Red de Popularización de la Ciencia y la Técnica en América Latina y el Caribe (Red POP) en Zacatecas. También se han impartido más de 3 o cursos de temas relacionados con la divulgación de la ciencia dirigidos a profesores y jóvenes divulgadores, incluidos tres a nivel internacional en República Dominicana (2009), Argentina (201I) y Bolivia (2013).
En 2007, en mancuerna con el Museo Universitario de Ciencias y con el apoyo de los Fondos Mixtos de Conacyt-Gobierno del Estado de Zacatecas, se creó la Sala Científica Móvil "Fantástica", que ha presentado $2 / 4$ exhibiciones de aprendizaje científico-lúdico, visitado 16 municipios del estado entre mayo de 2007 y junio de 2009 y capacitado a ig 8 guías que atendieron a más de 3o,ooo personas.

Quark también tiene a su cargo el Club "Astropatos". El club comenzó en 2008 y se dedica a realizar actividades de astronomía para aficionados mediante sesiones de observación, talleres, conferencias, campamentos y exposiciones. Con apoyo de Conacyt, en 20I3 se creó la "Caja de Aventuras Científicas", un kit didáctico que se está usando en ro entidades del país y que ha servido para coordinar acciones de una red de grupos de talleres de divulgación de nuevas tecnologías.

Lo anterior muestra el impacto del grupo en diversos ámbitos de la divulgación. El Grupo Quark funciona, y funciona bien. Los frutos del trabajo lo han posicionado como un referente de las actividades de ciencia recreativa en México. Por lo mismo, es importante identificar cuáles son los factores que le permitieron pasar de un proyecto de comunicación pública de la ciencia viable a uno que, luego de i2 años de trabajo, puede calificarse como exitoso. 


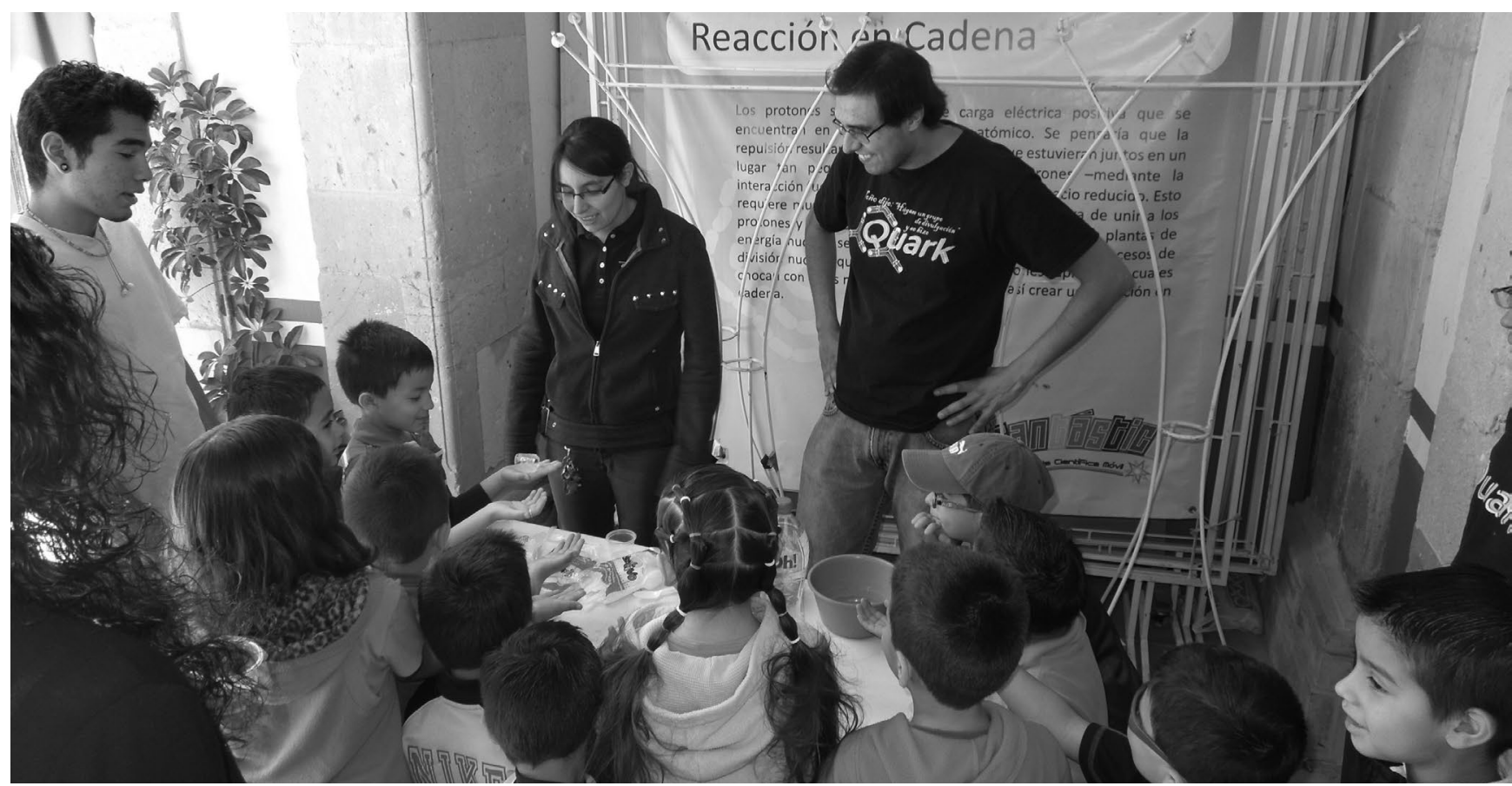

El club infantil del Grupo Quark trabaja los sábados en las instalaciones del Museo de Ciencias de la UAZ. La realización de experimentos es una de las modalidades para mostrar las aplicaciones científico-tecnológicas.

Foto: Grupo Quark.

\section{LAS FASES DE LOS VOLUNTARIOS}

El primer elemento que resalta es que la mayoría de los miembros de Quark son voluntarios, no reciben remuneración. Aún así, es uno de los grupos de divulgación con mayor número de integrantes en América Latina y el más grande dedicado a los talleres. Es importante discutir el proceso que permite atraer nuevos recursos humanos y prepararlos para desempeñarse como divulgadores.

\section{Fase 1. Atracción lúdica}

Estar en Quark es divertido. Ésta es una premisa que estuvo presente desde la fundación del grupo y ha sido desde entonces su directriz. Al Grupo Quark se puede llegar por muchos caminos distintos - amigos, invitación durante un taller u otras actividades, o por simple curiosidad-, pero todos los integrantes permanecen por un motivo, les gusta lo que se hace y cómo se hace.

Quark se distingue por el diseño de actividades lúdicas de divulgación. El disfrute personal es un punto de énfasis, casi la única obligación tajante impuesta por el grupo. La comprensión rara vez ocurre sin motivación para aprender; sin emoción y motivación nadie elegirá libremente involucrarse en aspectos más profundos de aprendizaje. ${ }^{2}$ Si el mismo divulgador no se divierte, es muy complicado que las personas involucradas en sus actividades lo hagan.

Precisamente, el deleite de los participantes es una de las armas más poderosas para reclutar nuevos integrantes de Quark. Es común encontrar jóvenes que encuentran las actividades tan agradables que no se conforman con ser usuarios, desean ser parte del equipo que las promueve. En este sentido, un caso particular de éxito se encuentra en el Club Infantil de la Ciencia, que se ha convertido en el semillero del Grupo Quark: en los últimos io años, 45 jóvenes del club han pasado al grupo, 2I de ellos siguen activos y cinco estudian en carreras de perfil científicotecnológico. Pero lo más trascendente es que, al menos en el núcleo de 8 o niños que participan en el Club Infantil de la Ciencia, se nota una visión trascendente: se forman personas que quieren crecer ligadas a la ciencia, en específico quieren ser divulgadores en Quark.

\section{Fase 2. Pertenencia y apropiación}

Yo soy de Quark en la misma medida que Quark es mío. Los integrantes se entienden a sí mismos como parte de un gran esfuerzo que al mismo tiempo les exige y pertenece. En función del grado de identidad alcanzado, establecen hasta dónde se comprometen con las 


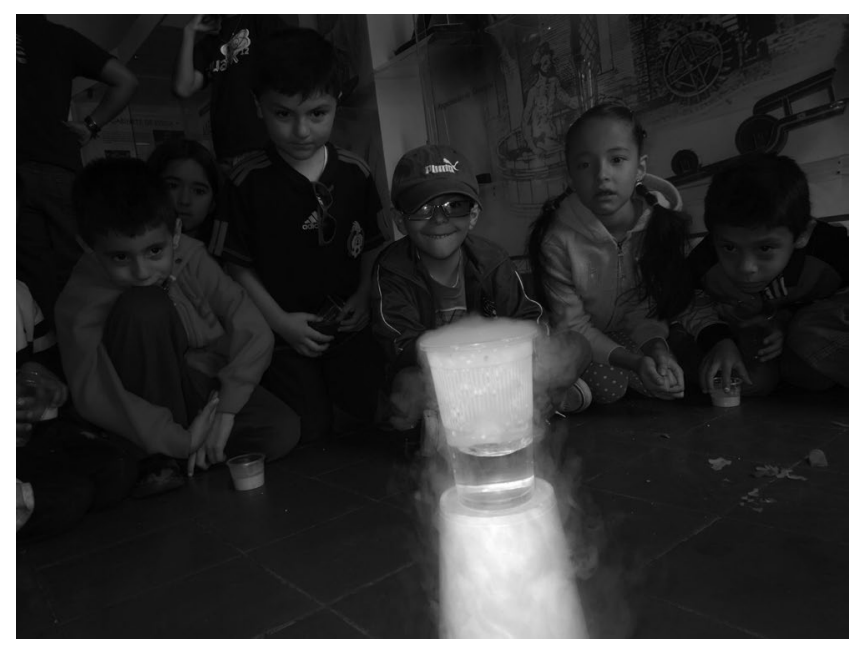

Develando los misterios de la ciencia. Foto: GRUPO QUARK.

acciones realizadas por el grupo. Este es, quizá, el primer filtro que de manera natural se aplica a los voluntarios. No se trata de uno excluyente, sino de un proceso que permite autodeterminar su grado de participación. Con el tiempo, algunos miembros comienzan a asumir mayores responsabilidades, que pueden ir desde ser encargado de un taller hasta asegurarse de tener los materiales listos, coordinar una sesión de talleres o, incluso, hacerse cargo de un programa de actividades.

En esta etapa cobran gran relevancia tres características indispensables de todo buen divulgador: voluntad, convicción y compromiso. El divulgador debe tener voluntad porque:

un divulgador no puede ni debe ser forzado a realizar sus actividades. La tarea de fomentar acercamientos entre ciencia y sociedad debe surgir de un gusto intrínseco por los procesos llevados a cabo [...] Todo divulgador debe ser un voluntario en su labor, independientemente de si ésta es o no remunerada. Es necesaria una determinación interna que nos estimule a hacer divulgación. ${ }^{3}$

Para ser un buen divulgador, lo primero es querer serlo, ya sea de manera transitoria o definitiva. Se puede ser voluntario sin ser profesional, pero para todo verdadero profesional su labor implica la voluntad de ser y hacer.

La convicción se refiere a la identidad entre el divulgador y los objetivos perseguidos por sus actividades; estar seguro de para qué se lleva a cabo el esfuerzo. Los divulgadores "[...] en resumidas cuentas,

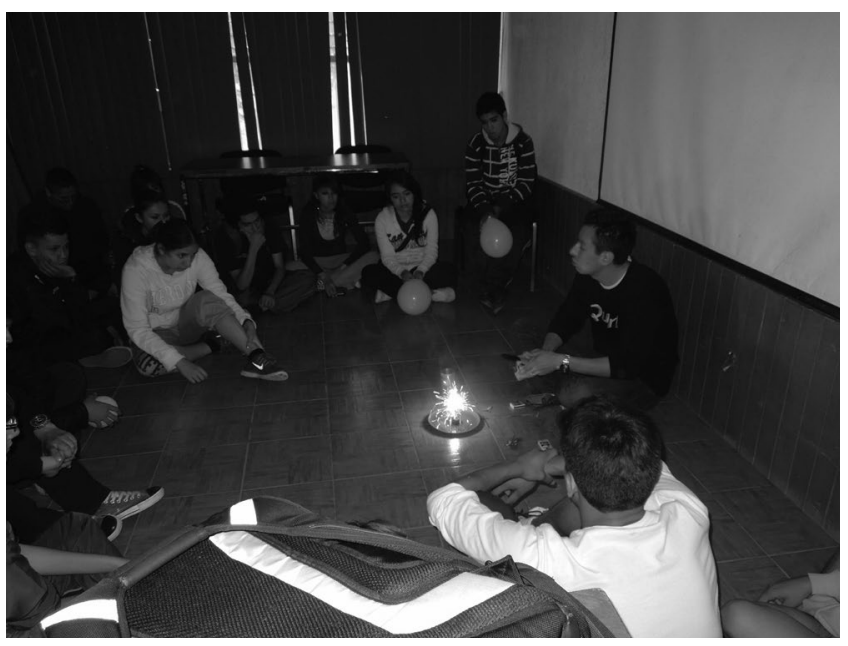

Las expresiones de la ciencia explicada a adolescentes.

Foto: Grupo Quark.

debemos ser los primeros en dimensionar de manera adecuada el valor de la divulgación". ${ }^{4}$

Finalmente, a partir de voluntad y convicción, se desarrolla un elemento fundamental e imprescindible para un buen trabajo de popularización científica: el compromiso. Se trata de la convicción de mejorar de manera constante, prepararse para ser mejores cada día, la motivación a "[...] estudiar todo el tiempo. Debemos de aprender de ciencia y de métodos de comunicación. Estudiar una disciplina nueva suele costar trabajo, pero conforme se avanza cada vez resulta más fácil".5

\section{Fase 3. Capacitación}

En Quark puedo aprender. Una inquietud recurrente entre quienes se incorporan a la labor del grupo es la falta de preparación. Algunos se preocupan por cómo llevar a cabo las actividades y otros tantos se interesan por fortalecer sus conocimientos sobre los temas que abordarán; hay también quienes desean trabajar para crecer en ambos aspectos.

Los cursos de capacitación representan un valor agregado para los participantes, ya sea como parte de la formación de aquellos que se dedicarán de lleno a la divulgación o como aporte al desarrollo de habilidades que les servirán sin importar su perfil profesional futuro. La capacitación se aplica de forma sistemática y se puede dividir, de modo general, en dos categorías distintas:

a) El abordaje teórico de los conceptos científicos discutidos en las actividades realizadas por Quark, que son impartidos por estudiantes o egresados de carreras 


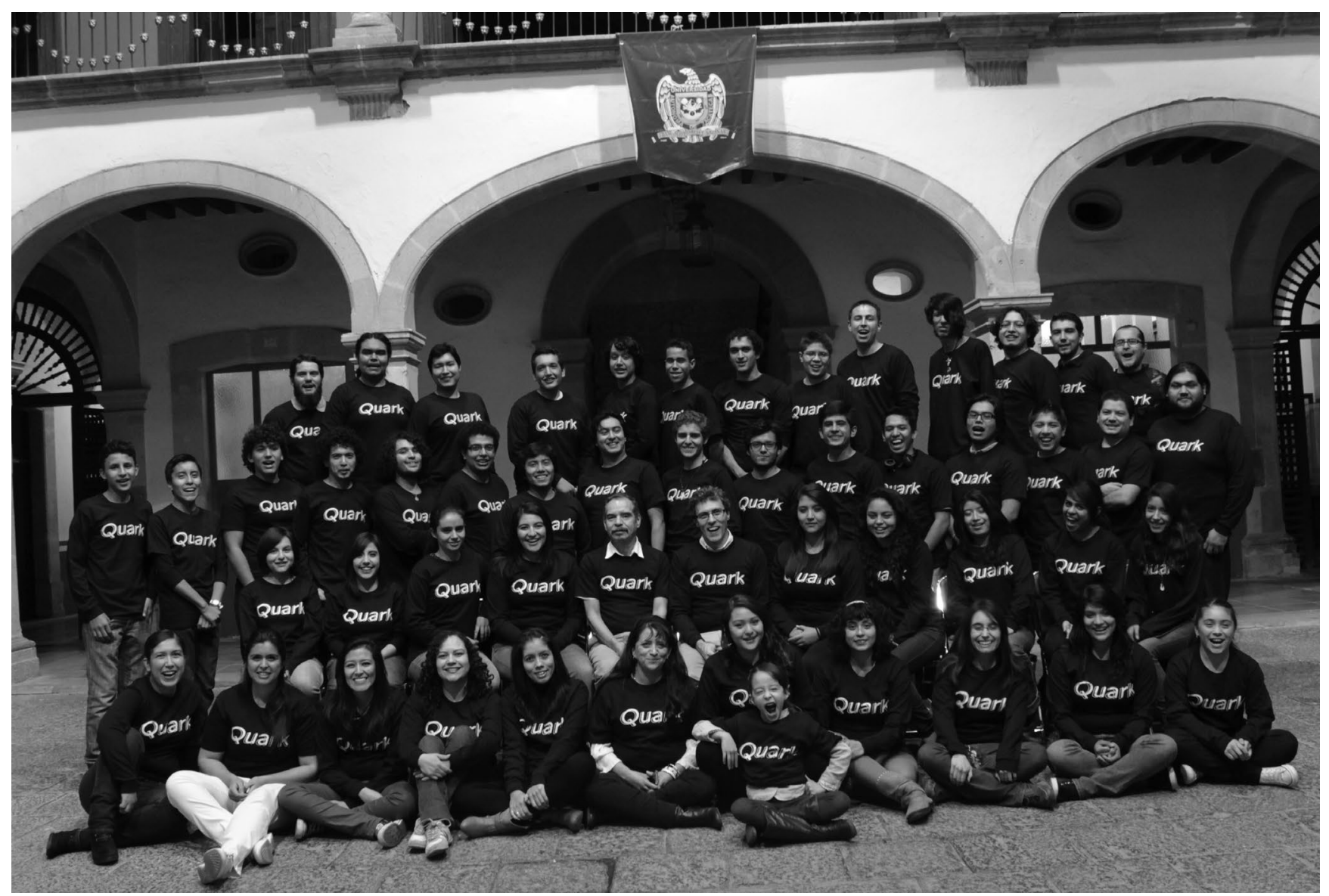

Jóvenes estudiantes voluntarios integran las filas del Grupo Quark. Poco a poco se forman como divulgadores de la ciencia y se abren camino en su ruta académica.

Foto: GRUPO QuARK.

afines. Para desarrollar las diferentes actividades, es necesario realizar constantes cursos de capacitación para que la mayor parte de los miembros obtenga el conocimiento adecuado para el manejo de los conceptos básicos indispensables de física, matemáticas, química y biología.

a) La revisión de los métodos y técnicas para desarrollar procesos de comunicación y divulgación, especialmente talleres, que están a cargo de algunos de los miembros más experimentados del grupo y de capacitadores externos. En este caso se revisan los principales elementos epistemológicos, pedagógicos y metodológicos que inciden en la mecánica de trabajo de Quark, primero en un curso intensivo de ocho semanas y posteriormente durante un año de trabajo supervisado por un integrante del grupo de reconocida experiencia. De esta forma se incorpora a todos los integrantes a las dinámicas utilizadas, a la vez que se aprovechan sus aportes para retroalimentarlas a través de procesos que complementan conocimientos tácitos y explícitos.
Fase 4. Profesionalización

Mira todo lo que se puede hacer. Esta es la etapa final y se distingue por lo que podría ser una extensión de la pertenencia y apropiación, una vez que se ha comprometido con su propia formación para construir los conocimientos y desarrollar las habilidades para ser un divulgador de calidad probada. Se está en posibilidad de impulsar formas de trabajo innovadoras y hasta nuevos programas para el grupo. Así, por ejemplo, han surgido el Club de Astronomía para "Astropatos", la iniciativa del Teatro Científico y las versiones más avanzadas del curso de capacitación. Todos encabezados por diferentes miembros de Quark.

En esta etapa también se pueden incluir los diversos trabajos académicos realizados acerca de la metodología de diversos aspectos de la divulgación. Los trabajos se socializan en eventos académicos dedicados a analizar las experiencias y dificultades encontradas durante el desarrollo de los programas llevados a cabo. Incluso el trabajo del grupo se ha publicado en cuatro 
libros: Ciencia en todos los rincones $(2009),{ }^{6}$ Para jugar con la ciencia (2011), Para jugar con la ciencia y la tecnología (20I3) y La ciencia en nuestras manos (2014).

Aunado a la capacitación interna, se han generado espacios de formación profesional consistentes en cursos y diplomados dirigidos principalmente a educadores y divulgadores ajenos a Grupo Quark. En 2005 y 20 Ir se llevaron a cabo diplomados en divulgación científica, se ha participado - como ponentes externos - en el diplomado en divulgación del Consejo Potosino de Ciencia y Tecnología, en varias ocasiones se ha colaborado con la Escuela Normal "Manuel Ávila Camacho" y cada año se imparten cursos en la Escuela Internacional de Verano de la UAZ.

\section{Conclusiones}

Es importante resaltar que las fases de un divulgador en Quark no son consecutivas sino acumulativas. Es decir, aun cuando se llega a ser profesional, la labor de divulgación sigue siendo divertida. Las diferentes etapas deben verse como una pirámide: es necesario establecer una sólida base con el fin de construir una cúspide más alta. Es en este sentido que todo profesio- nal - en cualquier actividad - debe ser un voluntario, de modo que disfrute lo que hace, asuma un compromiso y se prepare para lograrlo.

Finalmente, resulta ingenuo esperar que todo aquel que se una al Grupo Quark podrá concluir por completo el proceso evolutivo que va de voluntario recreativo, u ocasional, a profesional. Es obvio que, ya sea por cuestiones de disponibilidad de tiempo, por tener intereses diferentes o simplemente porque no forma parte de su plan de vida, algunos individuos llegarán sólo hasta cierta fase. Esto no debe leerse como un indicador negativo, sino como lo que es: el reflejo de un proceso de selección que se da de forma natural en todo desarrollo humano. Por el contrario, se debe considerar que el del Grupo Quark representa un método efectivo para atraer gente con el perfil deseado y propiciar su desarrollo profesional.

En el contexto social actual, la generación de profesionales de la divulgación científica es un asunto urgente. En nuestro país aún son pocas las oportunidades para prepararse como tal, y las ofertas de empleo son escasas también. Sin embargo, no podemos esperar que estas condiciones cambien por sí mismas, debemos ser proactivos y crear ambientes propicios para nosotros, allanando el camino para futuras generaciones.

\section{- notas $\cdot-$}

'Miguel Alcíbar Cuello (2004), "La divulgación mediática de la ciencia y la tecnología como recontextualización discursiva", Anàlisi: Quaderns de comunicació i cultura (3i), p. 45 .

"Bruce Lewenstein (2013), "Recreation in the Public Communication of Science and Technology", 89-IOI, en Reynoso et al., La recreación para la re-creación del conocimiento, Zacatecas, Somedicyt, p. $9^{2}$.
${ }^{3}$ Miguel García (2010), Los talleres de divulgación científica como agentes para el desarrollo de una cultura científica, Buenos Aires, Universidad de Quilmes.

údem.

5ulieta Fierro (2002), "La divulgación de la ciencia", en Juan Tonda et al., Antología de la divulgación de la ciencia en México, México, UNAM.

${ }^{6}$ Ídem. 


\section{SEPTIEMBRE}

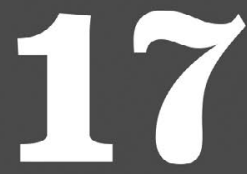

2014

10 A 15 HRS

SEMINARIO

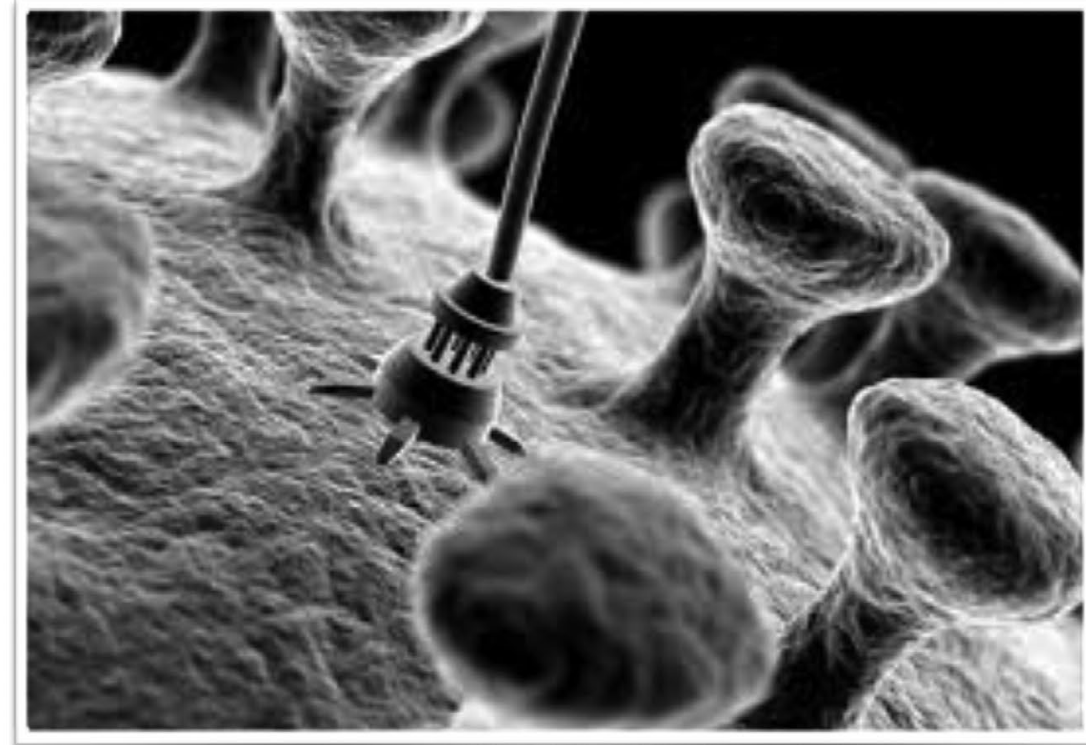

NANOTECNOLOGÍA \& SALUD: NUEVOS RETOS

$$
\stackrel{\star \star}{\star \star \star \star \star ~}_{\star \star}^{\star}
$$

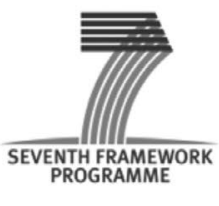

PROGRAMME
PROGRAMEWORK
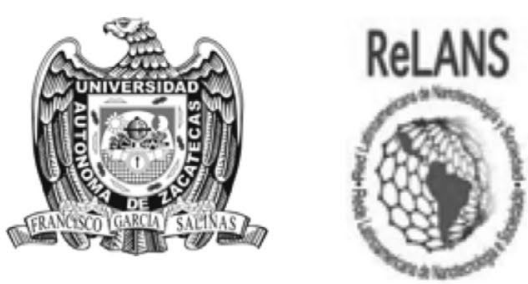

ESTUDIOS DEL DESARROLLO

Auditorio de la Unidad Académica en Estudios del Desarrollo, UAY. Participan:

Dndo. David Fajardo-Ortíz (Fac. Medicina, UNAM)

Dr. Eduardo Robles-Belmont (IIMAS, UNAM)

Dr. Alejandro Aguilera Galaviz (IyP, UAZ)

Dr. Pascual García Zamora (Maestría Ciencias de la Salud, UAZ)

Dndo. Miguel García Guerrero (Unidad Académica en Estudios del Desarrollo, UAZ)

Dnda. Laura Vidal Correa (Unidad Académica en Estudios del Desarrollo, UAZ)

Dr. Guillermo Foladori (Unidad Académica en Estudios del Desarrollo, UAZ)

Dr. Edgar Záyago Lau (Unidad Académica en Estudios del Desarrollo, UAZ)

Se entregarán certificados de asistencia 